\title{
ON THE COMBINATION OF NON-LOXODROMIC SUBSTITUTIONS*
}

\author{
BY \\ EDWARD B. VAN VLECK
}

In a brief but interesting communication on Finite groups of linear substitutions $\dagger$ Vivanti notes that a necessary condition that the product of two elliptic substitutions

$$
z^{\prime}=\frac{a z+b}{c z+d}
$$

be elliptic is that their four poles shall lie upon a common circle. Finding this to be precisely the necessary and sufficient condition that their product be non-loxodromic, I was led to the consideration of the necessary and sufficient condition that the product of any two non-loxodromic substitutions of given character-i.e., hyperbolic, parabolic, or elliptic-should be a non-loxodromic substitution, or, more restrictedly, one of specified character. The results are of very simple nature and make a sort of geometric multiplication table for non-loxodromic substitutions with non-loxodromic products.

The sufficiency of the conditions given for non-loxodromic products is involved in the structure of the familiar non-loxodromic groups, and it is probable also that their necessity, though not stated, is well known to many workers in the field of automorphic functions. It does not appear, however, that a systematic study of the combination of non-loxodromic substitutions and of the specific nature of their product has been previously made, and various facts brought out seem to be altogether new. Special reference may be made to the criterion given in Theorem 4 for the character of the nonloxodromic product. The combination of the substitutions affords a basis for the formation of non-loxodromic groups by synthesis. $\ddagger$

\section{Preliminary considerations}

In the following paper we shall consider exclusively non-loxodromic substitutions

$$
z^{\prime}=\frac{a z+b}{c z+d}
$$

* Presented to the Society, April, 1916.

† Rendiconti del Circolo di Palermo, vol. 35 (1913), p. 160.

† A rapid analytic derivation of these groups is given in Fubini's Introduzioni alla teoria dei gruppi discontinui e delle funzioni automorfe, pp. 189-193. His treatment is closer to that of the following pages than anything else that $I$ have found. 
These we shall assume to be always taken in unimodular form with determinant $\Delta \equiv a d-b c$ equal to 1 . Then the necessary and sufficient condition that $S$ shall be non-loxodromic is that $a+d$ shall be real. It is hyperbolic, parabolic, or elliptic according as $|a+d|$ is greater than, equal to, or less than 2.

The character of $S$ is not affected when it is transformed into $T^{-1} S T$ through any other linear substitution $T$. Any substitution with two distinct poles may be transformed into the form*

$$
z^{\prime}=k^{2} z
$$

For a hyperbolic substitution $k$ is real, while for an elliptic substitution it is an imaginary $e^{2 i \phi}$ of unit modulus. Let now $S_{1}$ be followed by any other non-loxodromic substitution $S$. Their product $S^{\prime \prime}=S S_{1}$ may be written in unimodular form

$$
z^{\prime}=\frac{a k z+b k^{-1}}{c k z+d k^{-1}},
$$

and will be non-loxodromic if $a k+d k^{-1}$ is real, its character depending upon $\left|a d+d k^{-1}\right|$. Since the same is true of $S_{1} S$, the character of the resulting substitution is independent of the order of the combination of $S$ and $S_{1}$.

Unless $c=0$, the poles of $S$ are given by

$$
z^{\prime}=\frac{(a-d) \pm \sqrt{(d+a)^{2}-4}}{2 c},
$$

those of $S^{\prime \prime}$ by

$$
z^{\prime}=\frac{\left(a k-d k^{-1}\right) \pm \sqrt{\left(d k^{-1}+a \overline{k)^{2}-4}\right.},}{2 c k},
$$

while those of $S_{1} S$ differ from (5) only by the substitution of $2 c k^{-1}$ for $2 c k$ in the denominator. When $c=0$, the substitutions $S, S_{1}$, and $S^{\prime \prime}$ have a common pole at infinity.

If our unimodular substitution (1) is real, it is necessarily non-loxodromic. Its two poles then lie upon the real axis if $|a+d| \geqq 2$, that is, when the substitution is hyperbolic or parabolic, while they will be symmetrically situated with respect to this axis when it is elliptic.

Suppose, conversely, that the poles of a hyperbolic or elliptic unimodular substitution are situated respectively in this manner. Then the radical in the numerator of (4) is either real or a pure imaginary, and therefore $a-d$ must be real to permit the poles of $S$ to have the assumed positions. Then $a, d$, and $c$ are real and hence the substitution must be real. If the substitution is parabolic, we must suppose in the converse, not merely that its pole lies upon the real axis, but also that this axis is an invariant curve of

${ }^{*}$ In unimodular form, $z^{\prime}=k z / k^{-1}$. 
the substitution. Then the four coefficients of the unimodular substitution are real except possibly for a common factor. As $a+d= \pm 2$, this factor must be real. Thus a unimodular substitution will be real if and only if it is a hyperbolic substitution with poles on the real axis, an elliptic substitution with poles symmetrically situated with respect to the axis, or a parabolic substitution having this axis as an invariant curve.

For the sake of completeness we will include the trivial case of the combination of the two non-loxodromic substitutions having a pole in common. The conditions under which they furnish a non-loxodromic product are seen at once after throwing this pole to infinity. The two substitutions then take the form (not necessarily unimodular)

$$
z^{\prime}=k^{2} z+a \quad\left(S_{3}\right), \quad z^{\prime}=k_{1}^{2} z+b \quad\left(S_{4}\right) .
$$

Obviously they generate a non-loxodromic product and group unless one substitution is elliptic and the other hyperbolic. In this group there is always included a parabolic substitution

$$
z^{\prime}=z+a\left(1-k_{1}^{2}\right)-b\left(1-k^{2}\right) \quad\left(S_{3} S_{4} S_{3}^{-1} S_{4}^{-1}\right) .
$$

This reduces to the identical substitution only if $a\left(1-k_{1}^{2}\right)=b\left(1-k^{2}\right)$, which is precisely the condition that $S_{3}$ and $S_{4}$ shall have their second pole in common.

These considerations make it clear that we have the following types of non-loxodromic groups with a single pole common to all the substitutions of the group, and only these types.

1. An elliptic-parabolic group of substitutions with one common pole.

2. A hyperbolic-parabolic group with one common pole.

3. A parabolic group whose substitutions have a common pole.

In addition, we have

4. Elliptic groups with both poles common.

5. Hyperbolic groups with both poles common.

There remains for consideration our main problem, that of the combination of two non-loxodromic substitutions without a common pole. If one is elliptic or hyperbolic, we may take it in the form (2) without loss of generality. Then $a+d$ and $a k+d k^{-1}$ are both real, provided the product of the two substitutions is non-loxodromic. Accordingly if $S_{1}$ is hyperbolic, $a$ and $d$ are real simultaneously with $k$. On the other hand, if $S_{1}$ is elliptic and accordingly $k$ an imaginary of unit modulus, $a$ and $d$ must be a pair of conjugate imaginaries. In either case, $a d$ and $b c$ are real.

\section{Combination with a hyperbolic substitution}

Take first for consideration the combination of a hyperbolic substitution (2) with another non-loxodromic substitution (1) so as to give a non-loxodromic 
product. As just stated, $k, a, d$, and $b c$ must be real. By a turn of axes $z=e^{i \theta} w$ we do not alter the substitution (2) and merely replace $b$ and $c$ in (1) by $b e^{-i \theta}$ and $c e^{i \theta}$. Hence we may make our substitution (1) take a real form. Then our two substitutions give rise to a group of real unimodular substitutions which are all non-loxodromic. Hence if $S$ is hyperbolic (parabolic), its poles (pole) lie with the poles 0 and $\infty$ of $S_{1}$ upon the real axis which is an invariant line, while they are symmetrically situated with respect to this axis if $S$ is elliptic. Conversel $\%$, by Section 1 , if these conditions are fulfilled, $S$ must be a real unimodular substitution, and hence generates with $S_{1}$ a non-loxodromic group of substitutions whose poles are disposed in similar fashion. When $S$ and $S_{1}$ are transformed through any linear substitution $T$, our result takes the following form.

THEOREM 1. The necessary and sufficient condition that the product of two hyperbolic substitutions be non-loxodromic is that their four poles shall be concyclic.* If a hyperbolic substitution is combined with a parabolic one, the corresponding condition is that the circle containing their three poles shall be invariant for the parabolic component. If a hyperbolic and elliptic substitution are combined, the condition for the formation of a non-loxodromic product is that there exists a circle upon which lie the poles of the hyperbolic component and with respect to which the poles of the elliptic component are symmetrically situated (i.e., are inverse points). In each case the group generated is non-loxodromic.

In combining our hyperbolic substitution $S_{1}$ with another non-loxodromic substitution the existence of a non-loxodromic product was sufficient, as noted, to ensure that the group generated should be non-loxodromic. When, however, it is combined with more than one non-loxodromic substitution, the non-loxodromic character of their simple products is no longer sufficient to ensure the non-loxodromic character of the entire group. Consider the combination of $S$ and $S_{1}$ with a third unimodular non-loxodromic substitution

$$
z^{\prime}=\frac{a^{\prime} z+b^{\prime}}{c^{\prime} z+d^{\prime}}
$$

so as to generate a non-loxodromic group. In Section 1 we disposed of the case in which all three substitutions have a pole in common. If $S_{1}$ has only one pole in common with $S$ and the other in common with $S^{\prime}$, it can not have the former pole in common with $S S^{\prime}$ since this pole would then belong also to $S^{-1}\left(S S^{\prime}\right)=S^{\prime}$, neither can it have its other pole in common with $S S^{\prime}$ since this pole would then belong also to $\left(S S^{\prime}\right) S^{\prime-1}=S$. Now $S S^{\prime}$ can be taken in place of either $S$ or $S^{\prime}$ as one of the generators of the group. It will be therefore no restriction to suppose that one of our two substitutions to be combined with $S_{1}$ has neither pole common with $S_{1}$. Let this substitution be $S$.

* The condition holds true when the poles are not distinct. See Section 1. 
As before, we will take $S$ in real form. Its combination with (6) gives a product

$$
z^{\prime}=\frac{\left(a a^{\prime}+b^{\prime} c\right) z+\left(a^{\prime} b+b^{\prime} d\right)}{\left(c^{\prime} a+d^{\prime} c\right) z+\left(b c^{\prime}+d d^{\prime}\right)}
$$

In both (6) and (7) the first and last coefficients must be real inasmuch as (6) and (7) by hypothesis combine with $S_{1}$ so as to produce non-loxodromic products. Consequently $b^{\prime} c$ and $b c^{\prime}$ are real. Now neither $b$ nor $c$ is zero inasmuch as the poles of $S_{1}$ and $S$ are distinct. It follows that $b^{\prime}, c^{\prime}$ are both real and hence the third substitution $S^{\prime}$ is real. The group generated by the three substitutions consists therefore of real unimodular substitutions which are all non-loxodromic.

Other non-loxodromic substitutions can clearly be added as generators of our non-loxodromic group provided that they also are real and unimodular. It has thus been shown that if a given set of non-loxodromic substitutions include a hyperbolic substitution (transformed above into form $S_{1}$ ), they will generate a non-loxodromic group if and only if they are capable of being transformed simultaneously into real unimodular form. In the italicized sentence of Section 1 the condition that a substitution should have such form was connected with the position of its poles relatively to the real axis. By transformation of our substitutions through any arbitrary substitution $T$ the real invariant axis becomes a circle. We obtain then the following result.

Theorem 2. If a set of non-loxodromic substitutions without a common pole includes at least one hyperbolic substitution, the necessary and sufficient condition that they generate a non-loxodromic group is the existence of a common invariant circle, with respect to which the poles of the elliptic generators must be symmetrically situated. Upon this circle lie the poles of the hyperbolic and parabolic generators.

It may be noted incidentally that this theorem admits all non-loxodromic substitutions which leave the circle invariant with the exception of elliptic substitutions of period 2 whose poles lie upon the circle.

Consider next the particular case of two hyperbolic substitutions (1) and (2) whose poles lie on a straight line through the origin and separate one another. A glance at (4) shows that the necessary and sufficient condition for this separation is

$$
\left|(a+d)^{2}-4\right|>|a-d|^{2} .
$$

Since $|a+d|>2$, this reduces to the condition $a d>1$ or its equivalent $b c>0$. Then

$$
\left|a k+d k^{-1}\right|>\left|a k+a^{-1} k^{-1}\right|>2,
$$

and the products $S^{\prime \prime}=S S_{1}$ and $S_{1} S$ are accordingly hyperbolic. Furthermore, the poles of $S^{\prime \prime}$ are separated by those of its first factor $S_{1}$ since 
$a k \cdot d k^{-1}>1$. For like reason the poles of $S^{\prime \prime-1}=S_{1}^{-1} S^{-1}$ are separated by those of $S^{-1}$. Hence the poles of $S^{\prime \prime}$ are separated by those of each of its factors. Thus we get the following theorem.

THEOREM 3. When the poles of two hyperbolic substitutions lie on a common circle and separate one another, the product of the two substitutions is hyperbolic, and its poles lie upon the same circle and are separated by those of each factor.

It may be pointed out that the group generated by the two substitutions will contain pairs of hyperbolic substitutions whose poles do not separate one another. In particular, the poles of $S S_{1}$ are not separated by those of $S_{1} S$ since they lie on opposite sides of the origin and differ by the positive factor $k^{-2}$ from the poles of $S_{1} S$.

Consider next the case of two hyperbolic substitutions whose poles lie vpon a commou ircle but do not separate one another. Their product may be either hyperbolic, parabolic, or elliptic. To determine which, I shall derive a general criterion for the character of the product of two non-loxodromic substitutions whose poles are distinct and which have a non-loxodromic product. Expressed in terms of its two poles $\gamma^{\prime}$ and $\delta^{\prime}$, our substitution (1) may be written

whence we have

$$
\frac{z^{\prime}-\gamma^{\prime}}{z^{\prime}-\delta^{\prime}}=k^{2}\left(\frac{z-\gamma^{\prime}}{z-\delta^{\prime}}\right),
$$

$$
z^{\prime}=\frac{\left(\gamma^{\prime}-\delta^{\prime} k^{\prime 2}\right) z+\gamma^{\prime} \delta^{\prime}\left(k^{2}-1\right)}{\left(1-k^{\prime 2}\right) z-\left(\delta^{\prime}-\gamma^{\prime} k^{\prime 2}\right)},
$$

in which the four coefficients are to be divided by $k^{\prime}\left(\gamma^{\prime}-\delta^{\prime}\right)$ in order to make the substitution unimodular. A comparison of (10) with (1) gives the values

$$
a=\frac{\gamma^{\prime}-\delta^{\prime} k^{\prime 2}}{k^{\prime}\left(\gamma^{\prime}-\delta^{\prime}\right)}, \quad d=-\frac{\left(\delta^{\prime}-\gamma^{\prime} k^{\prime 2}\right)}{k^{\prime}\left(\gamma^{\prime}-\delta^{\prime}\right)} .
$$

Since the poles $\gamma, \delta$ of $S_{1}$ have the special position $0, \infty$, the ratio $\gamma^{\prime} / \delta^{\prime}$ which appears in these expressions is the anharmonic ratio

$$
A=\left(\gamma^{\prime} \delta^{\prime} \gamma \delta\right)
$$

of the four poles of $S$ and $S_{1}$. If now these values of $a$ and $d$ are substituted in the inequalities

$$
\left|a k+d k^{-1}\right| \gtreqless 2
$$

determining the character of the product $S^{\prime \prime}=S S_{1}$, the inequalities may be written in the form (11) below. Now neither the anharmonic ratio $A$ nor the characteristic multipliers $k^{2}, k^{\prime 2}$ of $S_{1}$ and $S$ are changed in value when $S$ and $S_{1}$ are transformed through any linear substitution $T$. We obtain therefore the following useful criterion for the character of the product transformation. 
Theorem 4. If the four poles of two non-loxodromic substitutions are distinct and their product is non-loxodromic, the product will be hyperbolic, parabolic, or elliptic according as

$$
\left|1+k^{2}{k^{\prime}}^{2}-A\left(k^{2}+k^{\prime 2}\right)\right| \gtreqless 2\left|k k^{\prime}\right| \cdot|1-A| .
$$

Here $A$ denotes the anharmonic ratio $\left(\gamma^{\prime} \delta^{\prime} \gamma \delta\right)$ of the poles $\gamma, \delta$ and $\gamma^{\prime}, \delta^{\prime}$ of the two substitutions, and $k^{2}, k^{\prime 2}$ are the corresponding characteristic multipliers of the substitutions (as indicated in (9)).

The anharmonic ratio of four points in the z-plane is real only when the four points are concyclic. Consider now the case of two hyperbolic substitutions with concyclic poles. The constants $k$ and $k^{\prime}$ are real, and $A$ is negative when the two pairs of poles separate one another. The upper sign will then hold in the inequality (11), and the product of the two hyperbolic substitutions is accordingly hyperbolic, as already stated in Theorem 3. Suppose, on the other hand, that $A$ is positive so that the two pairs of poles do not separate one another. The sign of equality in (11) will hold if

that is, when

$$
1+k^{2} k^{\prime 2}-A\left(k^{2}+k^{\prime 2}\right)= \pm 2 k k^{\prime}(1-A),
$$

$$
A=\frac{\left(1+k k^{\prime}\right)^{2}}{\left(k+k^{\prime}\right)^{2}} \quad \text { or } \quad A=\frac{\left(1-k k^{\prime}\right)^{2}}{\left(k-k^{\prime}\right)^{2}} .
$$

The product of the two hyperbolic substitutions is then parabolic. It is easy to see that when $A$ lies between these two positive values the product is elliptic, while if $A$ is positive and does not lie between these limits, the product is hyperbolic. In the latter case the poles of the product $S^{\prime \prime}=S S_{1}$ or of $S^{\prime \prime \prime}=S_{1} S$ can not be separated by the poles of either of its factors. For if they were separated by those of $S$, the application of Theorem 3 to $S_{1}=S^{-1} S^{\prime \prime}$ and $S_{1}=S^{\prime \prime \prime} S^{-1}$ respectively would make the poles of $S$ and $S_{1}$ separate one another, contrary to hypothesis. For like reason they can not be separated by the poles of $S_{1}$.

Consider lastly the combination of a hyperbolic substitution $S_{1}$ with an elliptic substitution $S$ whose poles $\gamma^{\prime}, \delta^{\prime}$ are taken to lie symmetrically with respect to the real axis (or any line through the origin) so as to furnish a nonloxodromic product. We then have $|A|=\left|\gamma^{\prime}\right| /\left|\delta^{\prime}\right|=1$, and clearly this condition is also sufficient for the existence of such a product. Hence the necessary and sufficient condition that an elliptic and hyperbolic substitution should combine to give a non-loxodromic product is that the anharmonic ratio $\left(\gamma^{\prime} \delta^{\prime} \gamma \delta\right)$ of their four poles should be of unit modulus.

\section{Combination with a parabolic substitution}

Consider next the combination of a parabolic substitution $P$ with any other non-loxodromic substitution $S$. Without loss of generality we may suppose 
the parabolic substitution to be taken in the form $z^{\prime}=z+h$ where $h$ is real. In our substitution (1) $a+d$ must be real, and $c$ can not be zero since the two substitutions would then have a common pole. The product $P S$ has the for $m$

$$
z^{\prime}=\frac{a z+b}{c z+d}+h=\frac{(a+c h) z+b+d h}{c z+d}
$$

and will be non-loxodromic only if $a+d+c h$ is real, that is, if $c$ is real. By taking $n$ sufficiently large we may make $|a+d+n c h|>2$, and the group generated by the two substitutions must therefore contain a hyperbolic substitution $H=P^{n} S$. Now $P S \equiv P^{-(n-1)} H$, and accordingly the product $P S$ will be non-loxodromic under the conditions of Theorem 1 applied to the combination of a hyperbolic substitution $H$ and the parabolic substitution $P^{-(n-1)}$. But since $P$ and $P^{n-1}$ have the same system of invariant circles, the conditions of the theorem are fulfilled by $P$ and $H$ (which generate the same group as $P$ and $S$ ) at the same time as by $P^{n-1}$ and $H$. Hence by Theorems 1 and 2, we have immediately the following result.

Theorem 5. If two non-loxodromic substitutions are without a common pole and one of them is parabolic, the necessary and sufficient condition that they shall have a non-loxodromic product is the existence of a common invariant circle, with the added requirement that the poles of the second substitution must be symmetrically situated with respect to the circle if it is elliptic. The two substitutions then generate a non-loxodromic group in which hyperbolic substitutions are necessarily included.

\section{Combination of elliptic substitutions}

It remains now only to consider the combination of two non-loxodromic substitutions (1) and (2) which are both elliptic. When they have a nonloxodromic product, $a$ and $d$ are conjugate imaginaries with $|a+d|<2$, as noted in Section 1, while $k=e^{i \phi}$. Accordingly the two terms in the numerator of (4) are pure imaginaries, and the poles of $S$ lie with the poles of $S_{1}$ on a straight line through the origin. Conversely, suppose that the four poles of the two substitutions lie on the same straight line, necessarily through the origin. Since $a+d$ is real and less than 2 in absolute value, the two terms in the numerator of (4) must both be pure imaginaries; hence $a$ and $d$ are conjugate imaginaries. Then $a k+d k^{-1}$ is real and the product of the two substitutions is non-loxodromic. Thus the necessary and sufficient condition for a non-loxodromic product is that their four poles shall lie on a straight line. By transformation of the two substitutions through an arbitrary substitution $T$ this becomes the requirement that the four poles shall be concyclic.

If $\left|a k+d k^{-1}\right|<2$, the product $S^{\prime \prime}=S S_{1}$ is elliptic. Inasmuch as the products $S^{\prime \prime} S_{1}^{-1}=S$ and $S^{-1} S^{\prime \prime}=S_{1}$ are elliptic, the poles of $S^{\prime \prime}$ must be 
concyclic with the poles of $S$, also with the poles of $S_{1}$. Now the two terms in the numerator of (5) as well as (4) are pure imaginaries, and a comparison of their denominators shows that the poles of $S^{\prime \prime}$ lie on a line through the origin which makes an angle $-\arg k=-\phi$ with the line containing the poles of $S_{1}$ (or $+\phi$ if the order of the two factors is reversed). When the product is parabolic, the two poles of $S^{\prime \prime}$ come together on this line. Lastly, if $\left|a k+d k^{-1}\right|>2$, the numerator of (5) has the form $i A \pm B$, so that the poles of the hyperbolic product $S^{\prime \prime}$ are symmetrically situated with respect to the same line.

These results give the following theorem.

Theorem 6. The necessary and sufficient condition that the product of two elliptic substitutions be non-loxodromic is that their four poles shall lie upon a common circle. When the product is elliptic, its two poles are concyclic with the poles of each factor, and the circle containing the poles of $S_{1}$ and $S^{\prime \prime}=S S_{1}$ makes an angle - $\phi$ with the circle containing the poles of $S_{1}$ and $S$, if the multiplier of $S_{1}$ is $k^{2}=e^{2 i \phi}$ (or $+\phi$, if the order of the two factors is reversed). The two poles of $S^{\prime \prime}$ come together on the former circle when $S^{\prime \prime}$ is parabolic and are symmetrically situated with respect to the circle when it is hyperbolic.

Two cases will now be distinguished according as the poles of $S$ and $S_{1}$ do or do not separate one another. The condition for the separation is given in (8). Since $a$ and $d$ are now conjugate imaginaries with $|a+d|<2$, this reduces to the inequality $a d<1$ or its equivalent $b c<0$. This makes $|a| \equiv|d|<1$ and $\left|a k+d k^{-1}\right|<2$, and therefore the product of the two substitutions is elliptic when their poles separate one another. Further, the poles $P_{1}^{\prime \prime}, P_{2}^{\prime \prime}$ of the elliptic product $S^{\prime \prime}$ by Theorem 6 must be concyclic with the poles $P_{1}, P_{2}$ of $S$, also with the poles 0 and $\infty$ of $S_{1}$ and hence lie on a straight line through the origin. Since, moreover, the poles of $S$ lie on a line through the origin and are separated by the origin, it follows geometrically that the poles of $S^{\prime \prime}$ are separated by the poles of $S_{1}$ and of $S$. We have now the following result.

Theorem 7. If the poles of two elliptic substitutions are concyclic and separate one anoiher, the product of the substitutions is also elliptic, and its poles are concyclic with the two poles of each factor and are separated by them.

We have now the equal negative segment-products, $O P_{1}^{\prime \prime} \cdot O P_{2}^{\prime \prime}=O P_{1} \cdot O P_{2}$. Consider now any elliptic substitutions $S$ and $S^{\prime \prime}$ whose two poles lie on a line through the origin, and on opposite sides in such a manner that the above segment prcducts are equal. Then its poles $P_{1}^{\prime \prime}, P_{2}^{\prime \prime}$ are concyclic with $P_{1}, P_{2}$ and are separated by the same. Consequently the product $S S^{\prime \prime}$ will be elliptic, its two poles $Q_{1}, Q_{2}$ being concyclic with the poles of $S$, also of $S^{\prime \prime}$ and separated by the same. It follows at once by simple geometry that $Q_{1}$ and $Q_{2}$ must lie on some line through the origin and be so situated on opposite sides of the 
origin that $O Q_{1} \cdot O Q_{2}=O P_{1} \cdot O P_{2}=O P_{1}^{\prime \prime} \cdot O P_{2}^{\prime \prime}$. Hence the continued product of such elliptic substitutions with one another and with $S_{1}$ can give rise only to elliptic substitutions with the same properties. Thus the group generated by $S$ and $S_{1}$ is composed only of elliptic substitutions.

Consider now any third non-loxodromic substitution which combines with our two elliptic substitutions $S$ and $S_{1}$ so as to generate a non-loxodromic

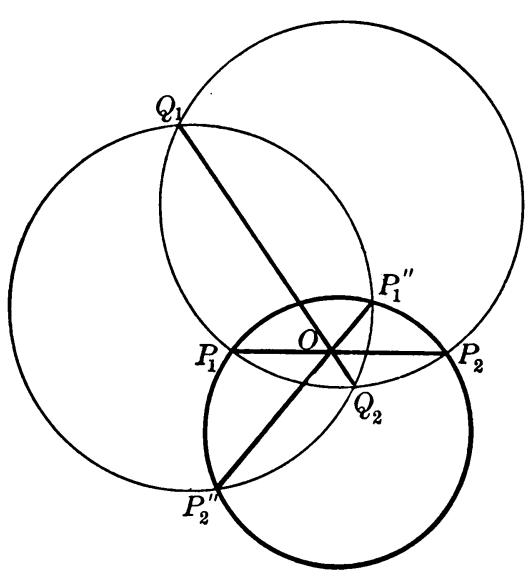
group. If this third substitution is hyperbolic, it cannot have a pole common with either $S$ or $S_{1}$ since their product would then be loxodromic (Section 1). But if the hyperbolic substitution has no pole common with either elliptic substitution, then by Theorem 2 the poles of the two elliptic substitutions must be pairs of inverse points with respect to a common circle. They therefore lie on an orthogonal crrcle without separating one another, which contradicts our hypothesis regarding $S$ and $S_{1}$. If, on the other hand, the third substitution is parabolic, its pole cannot be a pole of both elliptic substitutions. Then, by Theorem 5 , there must be a hyperbolic substitution in the group generated, a possibility which has just been excluded. It follows that the third substitution must be elliptic. If elliptic, its pores $P_{1}^{\prime}, P_{2}^{\prime}$ must be concyclic with the poles of $S$, also with the poles of $S_{1}$ and hence lying on a straight line through the origin. Then, like the poles of $S$, they must lie on opposite sides of the origin and be so situated that $O P_{1}^{\prime} \cdot O P_{2}^{\prime}=O P_{1} \cdot O P_{2}$. The considerations of the preceding paragraph then apply. We thus arrive at the following theorem.

Theorem 8. If the poles of two given elliptic substitutions are concyclic and separate one another, any other non-loxodromic substitution will combine with them so as to generate a non-loxodromic group if, and only if, it is an elliptic substituition whose poles are concyclic with the two poles of each of the two given substitutions. Any number of such substitutions will combine with one another and with the two given substitutions so as to give an elliptic group, and the poles of any two substitutions of the group are concyclic and separate one another.

By forming the product of the two values of $z$ in (4) we find

$$
\overline{O P}_{1} \cdot \overline{O P}_{2}=|b| /|c|,
$$

the value of which was seen to be the same for all substitutions of our elliptic group containing $S_{1}$. Since also $b \neq 0$ and $c \neq 0$, it is possible by a change of variable $w=m z$ to make simultaneously $|b|=|c|$ in all substitutions of our elliptic group. The change does not affect the values of $a$ and $d$ nor 
destroy the unimodular character of the substitutions. Since in each substitution $a$ and $d$ are conjugate imaginaries and $b c=a d-1<0$, we may place

$$
a=e+f i, \quad d=e-f i, \quad b=g+h i, \quad-c=g-h i .
$$

The substitutions of the group then take the canonical form

$$
z^{\prime}=\frac{(e+f i) z+g+h i}{-(g-h i) z+e-f i},
$$

where $e^{2}+f^{2}+g^{2}+h^{2}=1$. This is the familiar form for the group of rotations of a sphere.

There remains for consideration the case of two elliptic substitutions (1) and (2) whose poles lie on a straight line through the origin without separating one another. These two substitutions leave invariant a circle around the origin, having a radius whose square is equal to $\overrightarrow{O P}_{1} \cdot \overrightarrow{O P}_{2}=|b| /|c|$. This is therefore invariant for the group which they generate. We might now proceed in a manner parallel to that for the case of two elliptic substitutions whose poles separate one another, but instead of doing so we shall establish that the group generated contains a hyperbolic substitution. For consider the product obtained by following the substitution

$$
z^{\prime}=\frac{d k^{-1} z-b k}{-c k^{-1} z+a k} \quad\left(S^{-1} S_{1}^{-1}\right)
$$

with the substitution (3). This has the form

$$
z^{\prime}=\frac{\left(a d-b c k^{-2}\right) z+a b\left(1-k^{2}\right)}{c d\left(1-k^{-2}\right) z+\left(a d-b c k^{2}\right)}, \quad\left(S S_{1} S^{-1} S^{-1}\right) .
$$

Here

$$
\left(a d-b c k^{-2}\right)+\left(a d-b c k^{2}\right)=2-b c\left(k-k^{-1}\right)^{2}>2,
$$

inasmuch as $b c$ is positive and $k-k^{-1}$ is a pure imaginary because $k=e^{i \phi}$. Accordingly (13) is a hyperbolic substitution, the poles of which lie upon the above invariant circle.

The poles of our two elliptic substitutions and of (13) fulfill the requirement of Theorem 2, and we have therefore the following result.

Theorem 9. When the poles of two elliptic substitutions lie upon a common circle without separating one another, they generate a non-loxodromic group in which hyperbolic substitutions are included.

An immediate corollary of this theorem is that if a group contains only a finite number of substitutions (necessarily elliptic), it must be a group of elliptic substitutions in which the poles of any pair are concyclic and separate one another. The group can therefore be transformed into the familiar form (12) of the group of rotations of a sphere. 
Another corollary is that the modular group* and, more generally, any group with a "principal circle" whose interior is transformed into itself by every substitution of the group, cannot contain any subgroups with a finite number of substitutions other than cyclical subgroups of elliptical substitutions. For, every such subgroup must be composed of elliptical substitutions whose points are symmetrically situated with respect to this circle. Now the poles of two such substitutions will not separate one another on their common circle; hence, unless all the elliptic substitutions all have their poles in common the group must contain a hyperbolic substitution and therefore an infinite number of substitutions. But a finite group of elliptic substitutions with common poles make a cyclic subgroup.

The non-loxodromic group generated by two elliptic substitutions whose poles do not separate one another can be transformed into a form corresponding to (12). The invariant circle of (1) and (2) is one with its center at the origin for which

$$
R^{2}=\overline{O P}_{1} \cdot \overline{O P}_{2}=|b| /|c| .
$$

Since the value of $\overline{O P}_{1} \cdot \overline{O P}_{2}$ is the same for all substitutions of the group, we may as before effect a change of variable $w=m z$ so as to make simultaneously $|b|=|c|$ for each substitution of the group. Since now $a$ and $d$ are conjugate imaginaries while $b c>0$, the group takes the form

$$
z^{\prime}=\frac{(e+f i) z+g+h i}{(g-h i) z+e-f i},
$$

where $e^{2}+f^{2}-g^{2}-h^{2}=1$. This formula may be regarded as a second canonical form for a non-loxodromic group of substitutions without a common pole which include a hyperbolic substitution. When the invariant or "principal" circle is transformed into the real axis, the group takes the real unimodular form which has been previously noted.

\section{NON-LOXODROMIC GROUPS. INTERPRETATION OF A GROUP WITH PRINCIPAL CIRCLE UPON A HYPERBOLOID OF TWO SHEETS}

In Section 1 we enumerated the kinds of non-loxodromic groups with a pole common to all the substitutions of the group. The possibilities for a group without a common pole were exhausted in the preceding paragraphs. We obtained

6. An elliptic group, which could be transformed into the canonical form of a rotation group.

* Theorem 9 brings out the intrinsic reason for the fact which does not appear in the special proof given by Fricke; cf: Vorlesungen ueber die Theorie der elliptischen Modulfunctionen, vol. 1, p. 309, footnote.

Vivanti, l.c., pp. 161-3, shows that when two elliptic substitutions belong to a finite group, their poles separate one another. 
All other possibilities are included under groups of substitutions which admit a common invariant circle. When this circle is transformed into the real axis, the substitutions take real unimodular form, and when into the unit circle, the form (14). It has been shown that unless all substitutions of the group have a common pole, a hyperbolic substitution is included. We have therefore the following four

Varieties of non-loxodromic groups with principal circle. (No common pole.)

7. A mixed elliptic, parabolic, hyperbolic group.

8. An elliptic-hyperbolic group.

An example of such a group is obtained by using as its generators unimodular substitutions (14) for which $f=h=0$ and substitutions for which $e=g=0$. The former are the hyperbolic substitutions of form (14) which have the poles \pm 1 , the latter are elliptic substitutions of period 2 which exchange the poles of the former. The combination of these substitutions give rise only to such substitutions. They are, in fact, the real substitutions of form (14).

9. A purely hyperbolic group.

10. A parabolic-hyperbolic group.

An example of a parabolic-hyperbolic group is afforded by the set of all unimodular substitutions (14) for which $f, g, h$ are even integers and $e$ is an odd integer. The inverse of such a substitution is of like character. In the product of two substitutions the four constants have the values

$$
\begin{array}{ll}
E=e e^{\prime}-f f^{\prime}+g g^{\prime}+h h^{\prime}, & G=e g^{\prime}+g e^{\prime}+f h^{\prime}-h f^{\prime}, \\
F=e f^{\prime}+f e^{\prime}+g h^{\prime}-h g^{\prime}, & H=e h^{\prime}+h e^{\prime}+g f^{\prime}-g^{\prime} f,
\end{array}
$$

and accordingly $E$ is odd and $F, G, H$ are even.

It is interesting to see the relation of this group to the modular group

$$
z^{\prime}=\frac{\alpha z+\beta}{\gamma z+\delta}, \quad(\alpha \delta-\beta \gamma=1),
$$

in which $\alpha, \beta, \gamma, \delta$ are real integers. Let the latter group be transformed through the substitution

$$
z^{\prime}=\frac{\frac{1}{\sqrt{2}}(w+i)}{\frac{1}{\sqrt{2}}(i w+1)}
$$

which converts the real axis of $z$ into the unit circle of the $w$-plane. Thereby the modular group is transformed into a group of form (14), in which

$e=(\alpha+\delta) / 2, \quad f=(\beta-\gamma) / 2, \quad g=(\beta+\gamma) / 2, \quad h=(\alpha-\delta) / 2$.

It thus appears that the substitutions (14) with integral values of $e, f, g, h$ 
are a subgroup of the transformed modular group, and the particular parabolichyperbolic group under consideration is a sub-group of this subgroup.

The groups 7-10, taken in form (14), have an interpretation on the hyperboloid of two sheets, $\zeta^{2}-\xi^{2}-\eta^{2}=1$, closely analogous to the interpretation of the elliptic group (12) as a rotational group of the sphere. Put

$$
x=\frac{\xi}{\zeta-1}, \quad y=\frac{\eta}{\zeta-1}, \quad z=x+i y=\frac{\xi+i \eta}{\zeta-1}
$$

and accordingly

$$
\frac{\zeta+1}{\zeta-1}=x^{2}+y^{2} \text {. }
$$

To every point in the $z$-plane there is made to correspond by these equations a point on the hyperboloid, and conversely. The unit circle of the z-plane is transformed into the circular section of the hyperboloid at infinity, and the groups (14) are therefore groups of motion of the hyperboloid into itself which leave the circular section at infinity unaltered. Further, to any circle (or straight-line) of the $z$-plane there corresponds a plane section of the hyperboloid, and conversely. Since any linear transformation of the $z$-plane converts circles into circles, it follows that the motion of the hyperboloid must be a collineation into itself. A hyperbolic substitution (14) leaves invariant the circles passing through its poles on the unit circle, and therefore the corresponding invariant sections of the hyperboloid are hyperbolas cut out by parallel planes passing through two invariant points of its circular section at infinity. When the substitution (14) is parabolic, the two invariant points come together, and the invariant sections along which the points of the hyperboloid move are parabolas cut out by parallel planes. Lastly, when the substitution (14) is elliptic, it is easy to see analytically that the invariant sections are ellipses cut out by a system of parallel planes.

UNIVERSITY OF WISCONSIN 